\title{
Design of Smartphone-based Wound Assessment System
}

\author{
Vidyashree Dalya \\ Research Student, \\ Electronics Engineering Department \\ AISSMS's Institute of Information Technology \\ Pune, India
}

\author{
D. K. Shedge, PhD \\ HOD \\ AISSMS's Institute of \\ Information Technology \\ Pune, India
}

\begin{abstract}
The physical condition that affects the way the human body processes sugar (glucose) is known as diabetes. It is an alarming issue and is one of the major causes to prolong human wounds. Wounds examination process involves an expertise and constant follow ups to check and recommend wound details. This is a costly process and troublesome in case of bed ridden patients. Various systems like MEdical Digital PHOtogrammetric System (MEDPHOS), Measurement of Area and Volume Instrument System (MAVIS) have been developed for wound assessment. The systems suffer from high cost and maintenance. Moreover, these also need an expertise to perform assessment. As smart phones have become the part and parcel of our lives,, this project attempts to make use of Smart phones with a highresolution digital camera, assessing wounds by analyzing images of chronic foot ulcers. In this paper, we have developed an android based smartphone based solution involving the patients or hospital nurses actively. Additionally, we have also developed an online "Experts review" system which would send the image analysis notifications to the user. The proposed system consist of an android-based PC application. The user captures the image and uploads the image to the database. The image is then processed against the K-shift algorithm. The inferences like the wound status are then sent to the user and the image is also sent for expert review. The experts then examine the image, and based on the status of the wounds, they suggest for any changes in the treatment. Our simulation results show that the wound detection using K-mean shift algorithms gave good accuracy of approximately $80 \%$. This shows that with this smart phone system provides promising accuracy for wound image analysis.
\end{abstract}

\section{General Terms}

Image analysis, feature extraction, wound analysis.

\section{Keywords}

Diabetes; android based smartphone; mean shift; image processing

\section{INTRODUCTION}

"Home health care is a rising industry in medical streams". Home health care, assisted living facilities, long term care are some of the basic requirements of the patients discharged from severe hospital care. Home care services include taking care of the patients in their residences. There are two main types of diabetes - type 1 diabetes and type 2 diabetes. In the UK there are approximately 100,000 patients with leg ulcers every year and often these are not unique episodes. Recurrence rates depend on the aetology of the ulcer and ranges between $26 \%$ and $69 \%$. Studies report that UK district nurses spend between 10 to $50 \%$ of their time treating ulcers, both in the community and also in hospitals where up to $8 \%$ of bedridden and chair-bound patients may develop pressure sores. The treatment of chronic wounds comes at a cost of $£ 500$ million per annum. $3.5 \%$ of all adults in the USA suffer from venous leg ulcers. In most situations, elderly patients are prone to leg ulcers and pressure sores, however, the onset of the disease has been reported in early adult life.

There are more than 100 treatment regimens known for healing chronic wounds, ranging from various ointments and different bandage types to topical treatments with honey and even fly larvae. These regimes, however, treat only the chronic wound itself and not the underlying condition that is the cause of it. These range from malfunctions such as venous deficiency and diabetes to the side effect of other disease treatments such as the application of steroids. In other words: the treatment of wounds is a highly specialized and complicated "art" combined with plenty of science which depends to a large degree on the experience and also intuition of wound healing specialists. As a result "trial and error" treatment is for many chronic wound patients the norm rather than the exception.

Today's Indian lifestyle has made Home health care mandatory. In India, hiring the nurses for home care is considered as a taboo. This calls for better home-based health care facilities. It is estimated that for an average individual, $80 \%$ of health care needs can be met in the home environment. This can result in improved and lower medical costs for the patients.

At the moment, the only objective way of establishing whether a chronic wound is responding to a particular treatment is to measure the size of the wound. As wounds heal very slowly (months and years rather than weeks are typical) these measurement methods have to be precise enough to objectively document the small changes between patient contacts with the clinician. This is becoming increasingly important as the readiness of patients to start litigation actions against their health care provider (as already endemic in the US) is more and more found in the UK and other European countries. As per law, anything that is not documented is considered as not done. As a result more and more wound treatment protocols now include the requirement to document wound healing progress over time. The problem is that existing techniques are inaccurate, cumbersome, slow and prone to errors.

This paper is preliminary succeeds on getting combination of service charges and delivery efficiency. Thus trying to make use of technology to set benchmarks. 


\section{LITERATURE SURVEY}

There are multiple wound assessment methods. The most inaccurate method involves measuring the width and lengths of the wound with a ruler and then applying a formula that assumes elliptical shape to the wound. The less inaccurate method is placing a sterile transparent sheet on the wound and tracing the wound. The sheet is placed on metric graph paper and by counting the squares the area is found. The most accurate method involves taking a photograph of the wound with a ruler or size marker in the picture. The image then gets transferred to a computer and evaluated by software. The method is accurate provided the wound is (a) photographed correctly (camera perpendicular to the wound), (b) the ruler or size marker are at the same distance from the camera as the wound and (c) the wound is flat and not curved.

All the above methods make contact with the wound and they can therefore be painful and carry the risk of infection.

Volume measurements by filling the wound either with a fast setting alginate paste or with saline. In both cases it is important to get the right amount of substance into the wound which requires skill experience and a lot of luck (alginate setting too fast or too slow thus flowing out, saline not contained between wound and covering 'cling film' sheet, saline not absorbed too rapidly by the wound). The volume is measured by weighing the alginate once it has set or, in the case of the saline, by administering it with a calibrated syringe. Again, the methods make contact with the wound and risk infection. On top of that they are very messy, especially when material which may be contaminated by puss or wound exudate escapes.

\section{EXISTING TECHNIQUES}

\subsection{Measurement of Area and Volume} Instruement(MAVIS)

It uses the "structured light"/stereo photogrammetric technique, where a set of stripes of light are projected onto the wound surface. A PC linked camera picks up the stripe image and from the stripe positions (more to the left=closer to the camera, more to the right=further away) it can work out a 3-D map of the wound area. This method is quite costly and the process is to be carried under supervision.

\subsection{MEdical Digital PHOtogrammatic $\operatorname{System}(M E D P H O S)$}

It employs recent techniques in machine vision and image processing. It consists of three cameras and a projector. The projector serves as a texture generator to compensate for the lack of natural object points on the wound. This reduces the cost, however, it still requires supervision.

\section{PROPOSED SYSTEM}

The proposed system has advantages over the existing system as it addresses the cost concerns, travelling cost and reduces patient trauma.

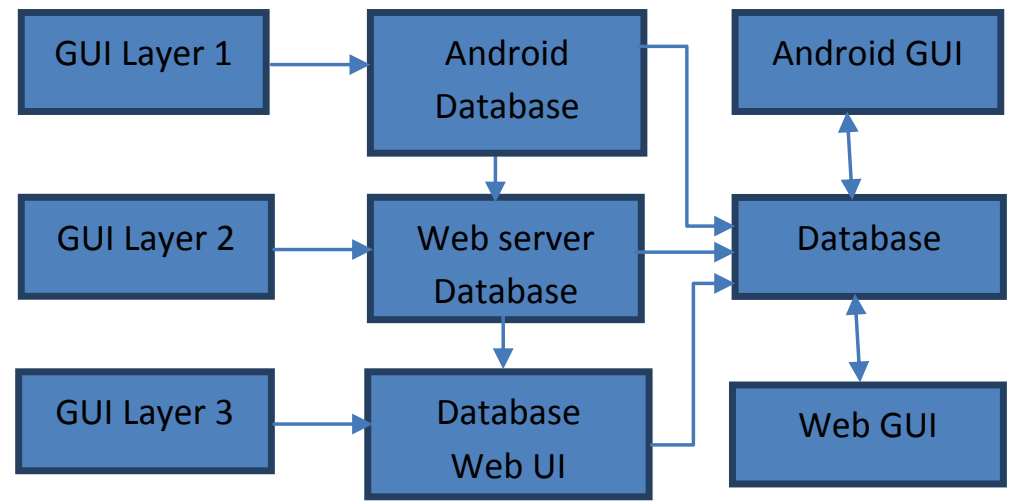

Fig 1: Proposed system Implementation.

\subsection{Software Interfaces}

Smartphone based diabetes app is developed under Android operating systems using the Java (JDK) and the android SDK (Software Development Kit) tools. The PC application is developed using Eclipse which is an open source community whose projects building tools and frameworks are used for creating general purpose application. Eclipse is used as a Java development environment. The centralized server must use MY-SQL database.

\subsection{Hardware Interfaces}

Smart phone based diabetes System will be developed as a PC application and mobile application. The mobile application is developed on Android platform and hence is solely supported on Android-powered devices. The android phone should have an inbuilt camera with capabilities of video recording.

The PC application is developed on Windows platform and hence is solely supported on Windows-powered PC's. The minimum configurations required for the $\mathrm{PC}$ application are

- $\quad 2.4 \mathrm{GHZ}, 80 \mathrm{~GB}$ HDD for installation.

- $\quad 512 \mathrm{MB}$ memory.

- Network Cards. 


\section{METHODOLOGY}

The methodology is shown in figure 2 .

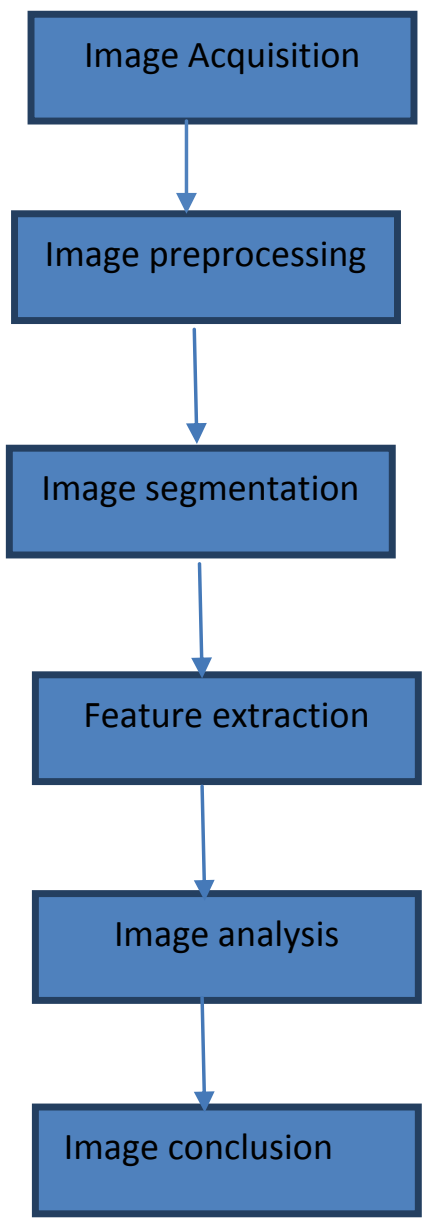

Fig 2: Flowchart of wound image analysis software.

\subsection{Image acquisition}

Firstly, the wound images are acquired using a digital camera with good resolution for better quality. The image database constructed for the application is responsible for the better efficiency of the classifier which in turn decides the robustness of the algorithm. Image compression is done by by using Ycbcr color space model.

\subsection{Image preprocessing}

In the second step, this image is pre-processed to suppress undesired distortions and enhance some image features giving a quantitative image data. The RGB images of wound are converted into Hue Saturation Intensity (HSI) color space representation. This is a standard process to specify colours in colour space presentation. As RGB is used for color generation and HSI for color descriptor. HSI model is an ideal tool for color perception. A pure color perceived by an observer is called as "hue".

\subsection{Image segmentation}

In Region based segmentation, the pixels of an object are grouped together. Thus we can easily identify the boundary. Once all the colour changes and textures are identified, the edge flow is converted into a vector.

In Edge based segmentation, segmentation identifies the boundaries and edges help to detect the image discontinuities.
Support Vector Machines (SVM) or adaptive algorithms carry out the classification.

In Threshold based segmentation, the threshold values of the histograms of the image help to perform image segmentation. The accuracy is high if the edge detection is done correctly. It is advantageous in terms of less computations involved. However, it is not accurate for complex images.

In feature based clustering, the images are clustered using $\mathrm{K}$ mean algorithm. It makes use of unsupervised Fuzzy $\mathrm{C}$ logic. The technique forms cylindrical decision elements of colour space and the image surface is detected by using threshold values of the histogram. The related pixels are clustered and the image is segmented and the colour components are changed if required. The difference between the image intensities and colour values also form an important characteristic of feature clustering. Virtual and real time clustering can be done. The image can then be refined and can be adjusted using Fuzzy logic. Clustering technique can be used for multispectral images making it different from other segmentation techniques.

\subsection{Feature extraction}

The next step is to extract the features of the captured images and then draw inferences of the images. The process classifies wound tissues within a wound as red, yellow, black or mixed tissues, which represent the different phases on the continuum of the wound healing process. Specifically, red tissues are viewed as the inflammatory (reaction) phase, regeneration, or maturation phase; yellow tissues imply infection or tissue containing slough that are not ready to heal; and black tissues indicate that the wound is not ready to heal. Based on the RYB wound evaluation model, wound analysis task is to classify all the pixels within the wound boundary into the RYB color categories and cluster them. Therefore, classical clustering methods can be applied to solve this task.

\subsection{Image analysis and conclusion}

The image is analyzed and is sent for expert review. The experts may then make various treatment decisions.

\section{RESULTS}

The results for the image analysis is done and shown the Figure 3. The input image is uploaded and its hue content is developed. Clustering is performed using thresholding. The final output image with mean shift is also shown here.

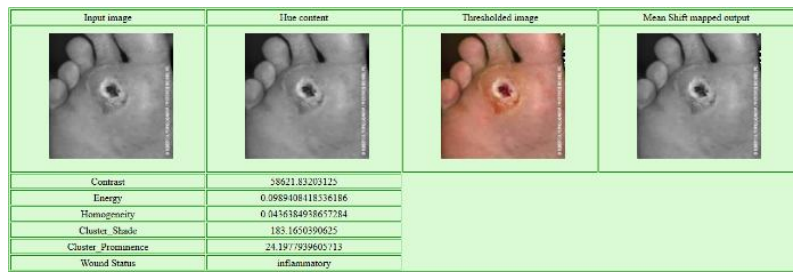

Fig 3: Wound image analysis using Mean shift algorithm.

The image analysis is performed by the experts and they document their findings and suggest the necessary changes in the treatment. The experts' reviews screen is shown in Figure 4. 


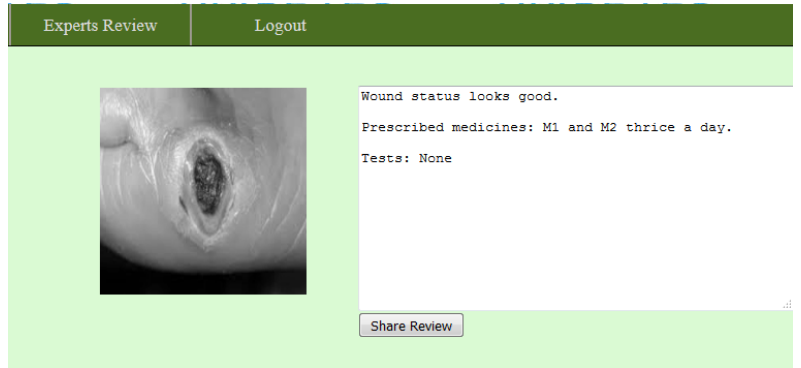

Fig 4: Wound image analysis performed by experts.

The patient receives the message and takes the appropriate action on the experts review comments as shown in figure 5 .

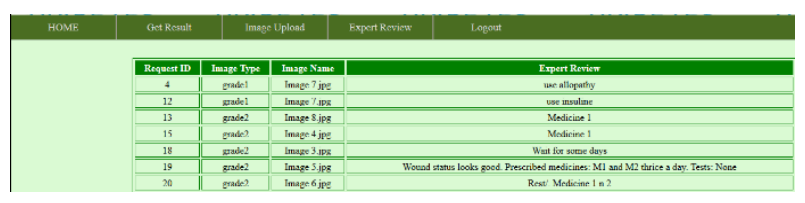

Fig 5: Patients message review screen

The experimental results are shown in Table 1 .

Table 1. Wound Image analysis results

\begin{tabular}{|c|c|c|c|}
\hline Image \# & $\begin{array}{c}\text { Cluster } \\
\text { shade }\end{array}$ & $\begin{array}{c}\text { Cluster } \\
\text { Prominence }\end{array}$ & $\begin{array}{c}\text { Wound } \\
\text { status }\end{array}$ \\
\hline 1 & 896.3994 & 135.7706 & Infection \\
\hline 2 & 183.1650 & 24.1977 & Inflammatory \\
\hline 3 & 216.5204 & 20.743 & $\begin{array}{c}\text { Necrotic } \\
\text { tissue state }\end{array}$ \\
\hline 4 & 240.4122 & 24.8955 & Infection \\
\hline
\end{tabular}

\section{CONCLUSIONS AND FUTURE WORK}

From the above discussion, it is concluded that a selfmanaged wound image analysis system for patients with type 2 diabetes suffering from foot ulcers is designed and implemented successfully. The mean-shift-based wound boundary determination algorithm provides a good accuracy, consistency and precision. The device setting allows one to obtain optimal resolution with ease and comfort. This product is an affordable device and can be purchased by patients or their caregivers, and in turn helps them to reduce both the frequency and the number of wound clinic visits.

In future work, the plan is to connect this system, from the smartphone to a server, which will allow more complex and computationally demanding wound boundary detection algorithms to be used. It is also planned to apply machine learning methods to achieve better boundary determination results with less restrictive assumptions. Furthermore, the aim is to compute a healing score to be assigned to each wound image to support trend analysis of a wound's healing status. Many a times some elderly patients may not be comfortable with operating a smartphone, but this concern could be addressed by further simplifying the image capture process to a simple voice command.

The next plan it to facilitate clinicians with remote access by creating cloud-based server support and creating a database to store the wound data for patients.

\section{REFERENCES}

[1] L. Wang, P.C. Pederson,D. Strong, B. Tulu and E. Agu,"Smart phone based wound assessment system for diabetic patients", presented at $13^{\text {th }}$ Diabetes Technology Meeting, San Francisco, CA, USA, Oct. 2013.

[2] Georgina Gethin (2006) the importance of coninous wound monitoring.

[3] V. Falanga, "The chronic wound: Impaired healing and solutions in context of wound bed preparation", Blood Cells Mol. Dis., vol. 32,no. 1, pp. 88-94,Jan 2004.

[4] Hazem Wannous, Yves Lucas,Sylvie Treuillet, "Efficient SVM Classifier based on colour and texture region features for wound tissue images".

[5] P. Plassman and T.D. Jones, "MAVIS: A non-invasive instrument to measurement of area and volume for wounds", Med. Eng. Phys.,vol. 20,no. 5,pp.332-338,Jul. 1998.

[6] A. Duarte, A. Sanchez, F. Fernandez, and A.S. Montemayor,'Improving image segmentation quality througheffective region merging using a heriarchical social metheuristic", Pattern Recog. Lett.,vol.27,pp.1239-1251,Aug.2006.

[7] A. Malian, A. Azizi, F. A. Van den Heuvel, and M. Zolfaghari, "Development of a robust photogrammetic system for monitoring the healing of bedscors", Photogrammetic Rec.,vol. 20,no. 111, pp. 241273,Jan.2005

[8] D. Pascale, RGB coordinates of the Macbeth Colour checker,BabelColourCompany,Montreal,Cubec,Canada.( 2006,Jun.).[Online]

[9] H. Wannous,Y. Lucas,Sylvie Treuillet, "Robust tissue classification for reproducible wound assessment in telemedicine environment,"J. Electron.Imag.,vol. 19,no. 2,pp. 023002-1-023002-9 